\title{
Efficacy and Resistance of ALK Inhibitors in Two Inflammatory Myofibroblastic Tumor Patients with ALK Fusions Assessed by Whole Exome and RNA Sequencing
}

This article was published in the following Dove Press journal: OncoTargets and Therapy

\author{
Chenlu Zhang' \\ Zhiming Wang' \\ Rongyuan Zhuang (1) \\ Xi Guo' \\ Yi Feng' \\ Feng Shen (iD)' \\ Wenshuai Liu ${ }^{2}$ \\ Yong Zhang ${ }^{3}$ \\ Hanxing Tong ${ }^{3}$ \\ Wending Sun ${ }^{4}$ \\ Jun Liu ${ }^{4}$ \\ Guan Wang ${ }^{4}$ \\ Chun $\mathrm{Dai}^{4}$ \\ Weiqi $\mathrm{Lu}^{3}$ \\ Yuhong Zhou' \\ 'Department of Medical Oncology, \\ Zhongshan Hospital, Fudan University, \\ Shanghai, People's Republic of China; \\ ${ }^{2}$ Department of General Surgery, \\ Shanghai Public Health Clinical Center, \\ Shanghai, People's Republic of China; \\ ${ }^{3}$ Department of General Surgery, \\ Zhongshan Hospital, Fudan University, \\ Shanghai, People's Republic of China; \\ ${ }^{4}$ GenomiCare Biotechnology Co. Ltd, \\ Shanghai, People's Republic of China
}

Correspondence: Weiqi Lu

Department of General Surgery, Zhongshan Hospital, Fudan University, I 80 Fenglin Road,

Shanghai, People's Republic of China

Email lu.weiqi@zs-hospital.sh.cn

Yuhong Zhou

Department of Medical Oncology,

Zhongshan Hospital, Fudan University, 180

Fenglin Road, Shanghai, People's Republic of

China

Email zhou.yuhong@zs-hospital.sh.cn

\begin{abstract}
We report two inflammatory myofibroblastic tumor (IMT) patients with ALK fusions (RRBP-ALK and TNS1-ALK, respectively). They both received tumor resection surgery and treatment with ALK inhibitors crizotinib followed by alectinib, and upon receiving each of the drugs, showed a brief response, then experienced recurrence or progression of the disease. During the treatment, whole exome sequencing (WES) and RNA sequencing (RNA-Seq) were applied to monitor potential drug-induced gene mutation and expression changes. A novel, secondary mutation in ALK exon 23 (L1196Q) was identified in patient 1 after alectinib resistance developed. Guided by this result, a newer ALK inhibitor, ceritinib was prescribed. The patient was able to achieve a partial response (PR) and is in good condition as of the manuscript date. On the contrary, there was no secondary mutation identified in ALK in patient 2 after drug resistance. While the expression of PTCH1, a negative regulator of the sonic hedgehog $(\mathrm{SHH})$ signaling pathway, was significantly reduced at the time after the treatment with crizotinib before that of alectinib. The expression of PTCH1 was also reduced after the treatment with alectinib. It was reported that ALK can exert its biological functions partially by activating SHH signaling pathway. The down-regulation of PTCH1 suggests the compensatory activation of SHH pathway may cause resistance to ALK inhibitors in IMT. Going forward, monitoring gene mutation and expression changes through DNA and RNA sequencing will be able to offer opportunities to investigate potential mechanisms of drug resistance and will help to achieve precise prescription for better treatment outcomes.
\end{abstract}

Keywords: inflammatory myofibroblastic tumor, crizotinib resistance, alectinib resistance, ALK L1196Q, sonic hedgehog pathway

\section{Introduction}

IMT is a rare mesenchymal neoplasm with characteristic histopathology. ${ }^{1}$ It is usually benign, but relapses in $25 \%$ and metastasizes in less than $2 \%$ patients. $^{2}$ Approximately $50 \%$ patients with IMT harbor ALK rearrangements in FISH tests. ${ }^{3}$ ALK rearrangements in tumors have conserved breakpoints in the introns preceding exons 19 or 20, therefore preserving the tyrosine kinase domain intact in the fusion products. ${ }^{4}$ At the same time, ALK fusion partners, such as ATIC, SEC31A, TPM4, TFG, RANBP2, CLTC, FN1, CARS and TPM3, 1,5,6 bring with them an oligomerization domain, which can support autophosphorylation and activation of the ALK kinase domain. ${ }^{7,8}$ 
Here, we report two metastatic/recurrent patients with ALK fusion. They both received crizotinib and alectinib treatment, responded for a brief period, but developed resistance to both ALK inhibitors. While the resistance can arise via multiple routes, the WES and RNA-Seq performed pre- and post-treatment have revealed some most likely mechanism of the resistance. Patient 1 acquired a secondary mutation (L1196Q) which likely changes the binding of crizotinib or alectinib with ALK so makes them ineffective. The patient was able to achieve a PR status after ceritinib treatment, suggesting ceritinib is effective for this particular mutation. Patient 2 had no secondary mutation in ALK after the use of crizotinib and alectinib. Gene expression analysis, however, revealed reduction of PTCH1 expression and activation of SHH pathway, suggesting pathway by-passing was possibly accounted for the resistance to the ALK inhibitors.

\section{Case Presentation Patient I}

A 22-year-old male was admitted to hospital with abdominal distension. Computed tomography (CT) demonstrated substantial ascites and diffuse peritoneal thickening (Figure 1A). Subsequently, IMT was diagnosed on January 30, 2018, based on the above information and pathological evaluation of the laparoscopic peritoneal biopsy (Figure 1H). Fluorescence in-situ hybridization (FISH) revealed a rearrangement in the ALK gene (Figure 1I). WES and RNA-Seq confirmed the $5^{\prime}$ end of ALK exon 21 was fused to the $3^{\prime}$ end of a $34 \mathrm{bp}$ piece of RRBP1 intron 21, creating a fusion gene consisted of RRBP1 exons 1-21 on the N-terminal and ALK exons 20-29 on the C-terminal (Figure 1J). The patient was started on systemic therapy of crizotinib at $250 \mathrm{mg}$ b.i.d. for 3 months. Following the treatment, the ascites subsided, and the abdominal distension and general condition of the patients were improved (Figure 1B). However, a follow-up CT scan on August 1, 2018 , showed a $\sim 2.3 \times 1.0 \mathrm{~cm}$ metastatic tumor mass behind the spleen and a small amount of peritoneal effusion (Figure 1C). Therefore, a second-generation ALK inhibitor alectinib at $600 \mathrm{mg}$ b.i.d. was prescribed. After two months, the disease was stable as shown in CT and the abdominal distension was relieved (Figure 1D). Still, a third CT scan on January 2, 2019, revealed PD with enlarged size of the tumor behind the spleen (Figure 1E). On January 17, 2019, the patient underwent surgery to excise the relapsing lesion. Tumor tissue was collected during the operation and analyzed in a second round of WES and RNA-Seq with the hope to identify the underlying cause of recurrence and assist in selection of subsequent treatment. A secondary mutation in ALK exon23, pL1196Q, was identified comparing to the first WES and RNA-Seq. The patient was continued on alectinib for another four months, until a new (4th) CT scan in May 2019 demonstrated a $5.1 \times 3.4 \mathrm{~cm}$ opacity at the porta hepatis and perisplenic space (Figure 1F). Another secondgeneration ALK inhibitor, ceritinib, was then administrated to the patient at $450 \mathrm{mg}$ daily starting from May 2019. He was able to achieve a PR (Figure 1G) and is under follow-up to this date.

\section{Patient 2}

The second patient was a 60 -year-old woman who initially complained pain in the right lower limb in March 2017 and was admitted to a local hospital. A CT scan revealed pelvic cavity occupation. A biopsy showed tissue necrosis and spindle-shaped cells in mild to moderate atypical patterns, highly suggesting leiomyosarcoma. Apatinib was prescribed to the patient for 5 months, but the tumor size grew. The patient was then transferred to our hospital. CT scan on January 23, 2018, displayed a mass of $\sim 6.6 \times$ $9.6 \mathrm{~cm}$ in the pelvic cavity invading to the right ureter and right iliac vessels, which caused severe hydronephrosis and renal failure (Figure 2A). Consequently, an urgent operation was performed on February 1, 2018, which included resection of the pelvic mass (including partial right ureter and the right iliac vein), exclusion of the right kidney, and installation of titanium marking clips near the surgical area. Tumor biopsies were collected during the operation and sequenced in April 2018. Parallel pathological examination showed hyper-proliferation (mitoses 38/50 HPF), spindle-shaped cells, necrosis and partial mucoid degeneration in the postoperative tissues (Figure 2I). The immunohistochemical (IHC) reading was $\mathrm{SMA}^{+}, \mathrm{Des}^{+}, \mathrm{ALK}^{+}$and $\mathrm{S}-100^{-}, \mathrm{Bcl}-2^{-}, \mathrm{CD} 117^{-}$. FISH and RNA-seq both indicated an ALK translocation event (Figure 2J and K). A 3 ' end fragment of ALK including exons 18-29 was fused to exon 18 of TNS1 therefore formed a TNS1-ALK translocation and the ALK kinase domain was preserved. Based on the above results, this patient was re-classified as IMT. Five months later, multiple recurrences and metastases were found in the right abdominal cavity, pelvic cavity and abdominal wall in CT imaging (Figure 2B and C). The patient was started with crizotinib at $250 \mathrm{mg}$ b.i.d. from July 2018. A follow-up exam in August 2018 showed tumor 


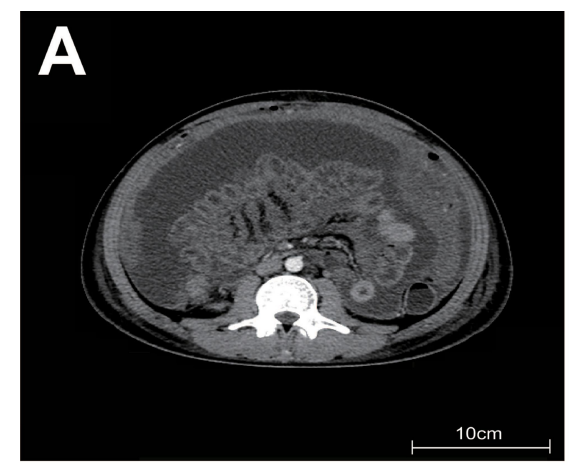

$01 / 15 / 2018$

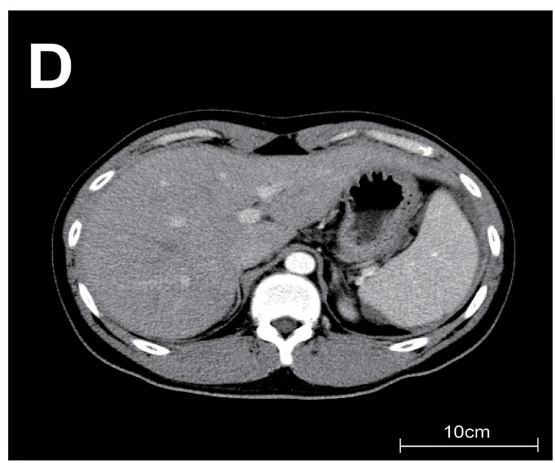

$11 / 05 / 2018$

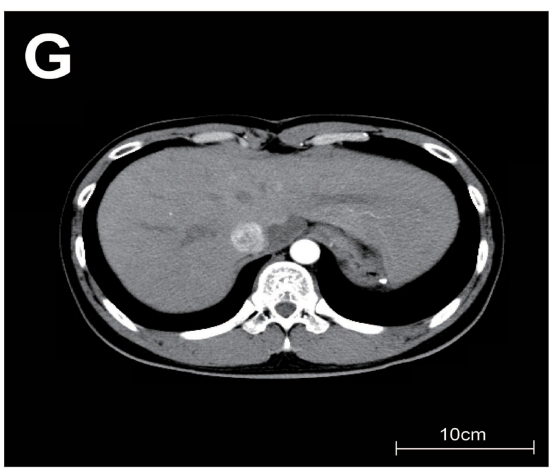

$10 / 12 / 2019$

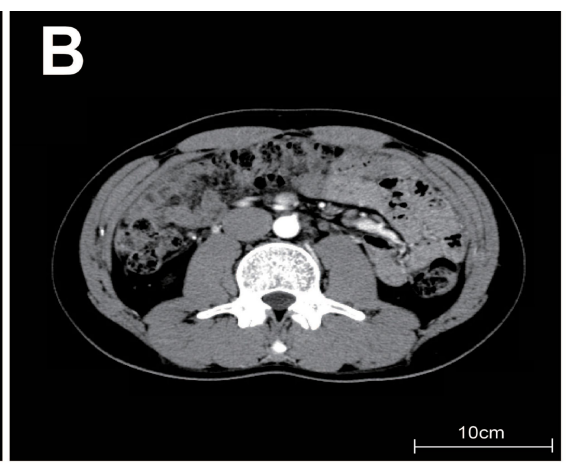

$05 / 22 / 2018$

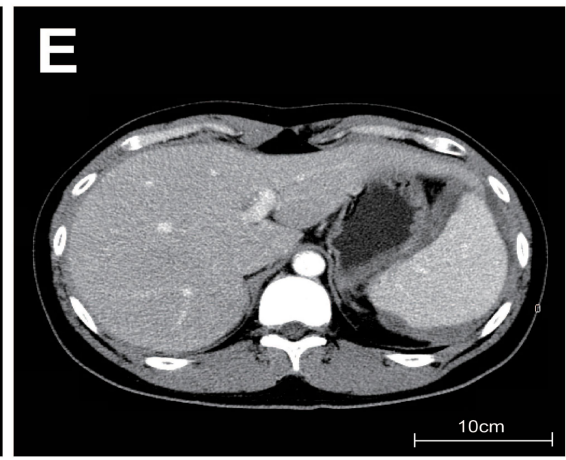

01/02/2019

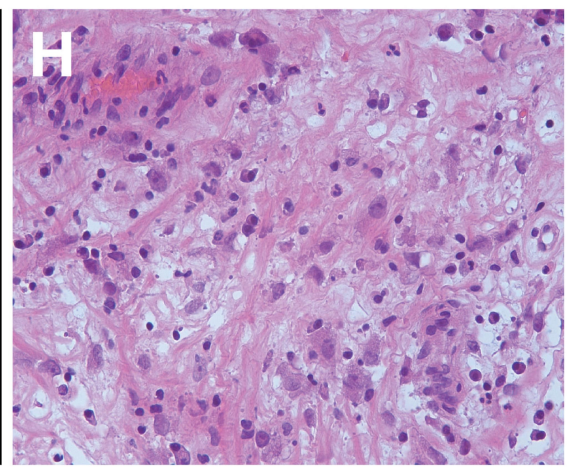

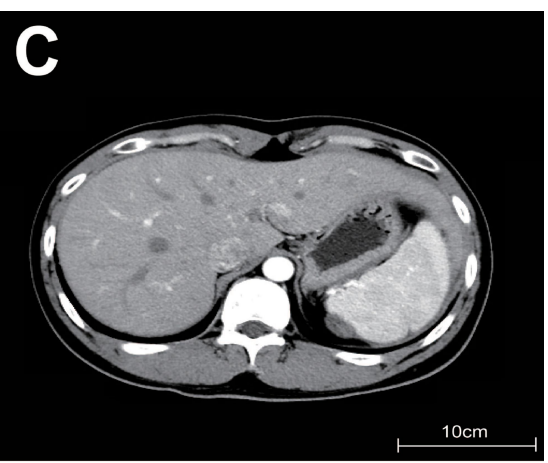

08/01/2018

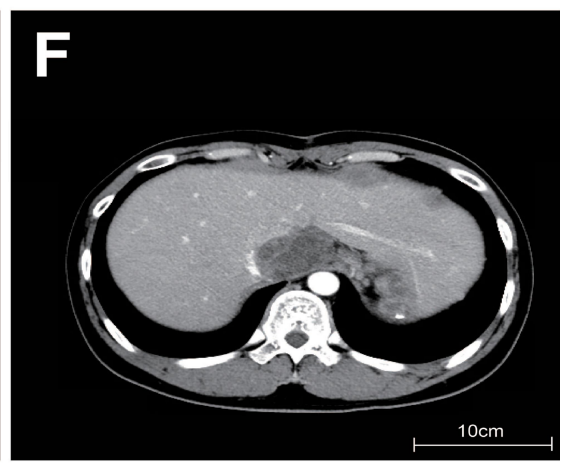

$05 / 16 / 2019$

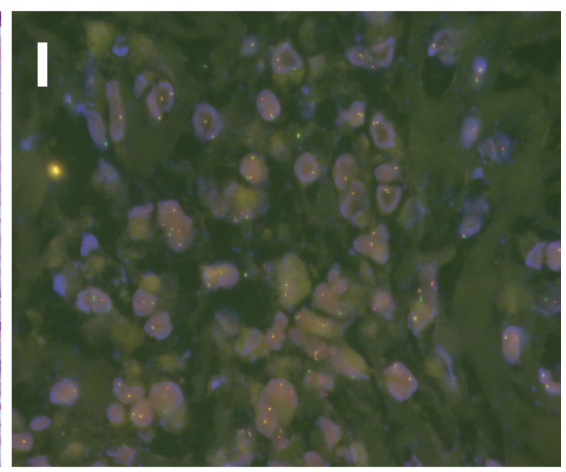

J

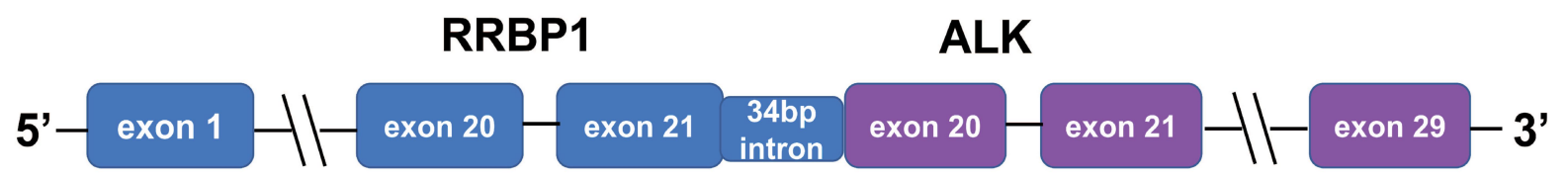

Figure I Radiological and histological evaluation and ALK translocation in patient I. (A-G) Chronological records of computed tomography (CT) images. The dates of the CTs were indicated below the images. (A) The baseline showed ascites and diffused peritoneal thickening with multiple focal masses and nodules. (B) The lesion was in partial regression after 3 months of crizotinib treatment. (C) A metastatic tumor mass of about $2.3 \times 1.0 \mathrm{~cm}$ behind the spleen and a small amount of peritoneal effusion were present when the patient became resistant to crizotinib. (D) The mass behind the spleen was stable after two months of alectinib treatment. (E) The tumor behind the spleen was enlarged again when it became resistant to alectinib. $(\mathbf{F})$ A recurrence lesion of $5.1 \times 3.4 \mathrm{~cm}$ at the porta hepatis and perisplenic space. (G) The tumor was relieved after receiving ceritinib. $(\mathbf{H})$ Micrograph of hematoxylin and eosin (HE) staining of the tumor biopsy at diagnosis (original magnification $\times 40$ ). (I) FISH image of the tumor biopsy at diagnosis labeled by an ALK probe (original magnification $\times 40$ ). (J) Diagram of ALK translocation. A $3^{\prime}$ end fragment of ALK starting from exon 20 is fused behind base 34 of RRBPI intron 21 . 


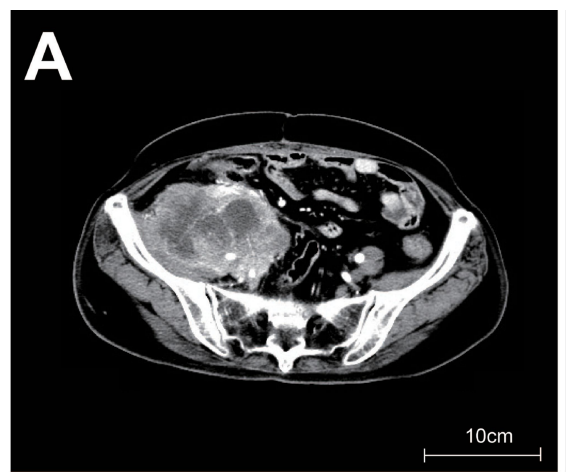

$01 / 23 / 2018$

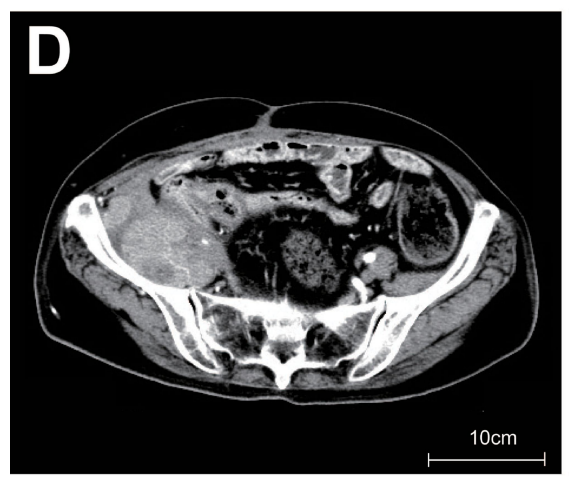

$08 / 16 / 2018$

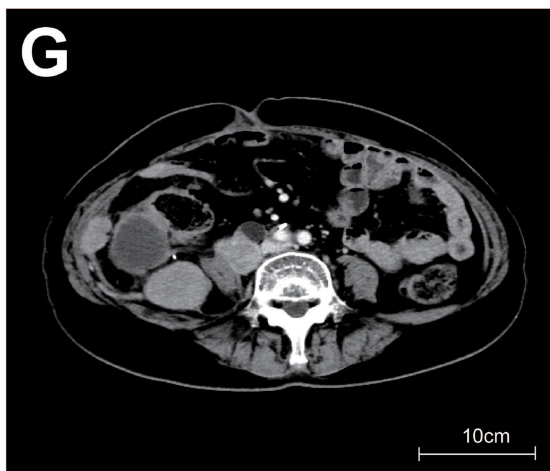

$11 / 30 / 2018$

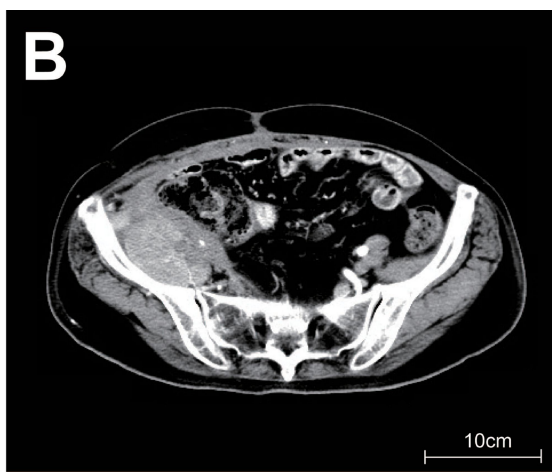

$07 / 18 / 2018$

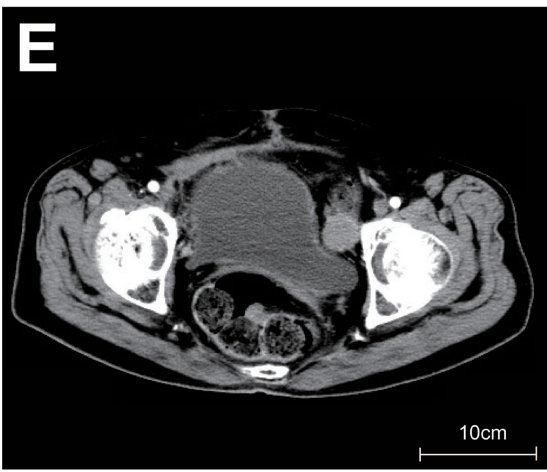

$08 / 16 / 2018$

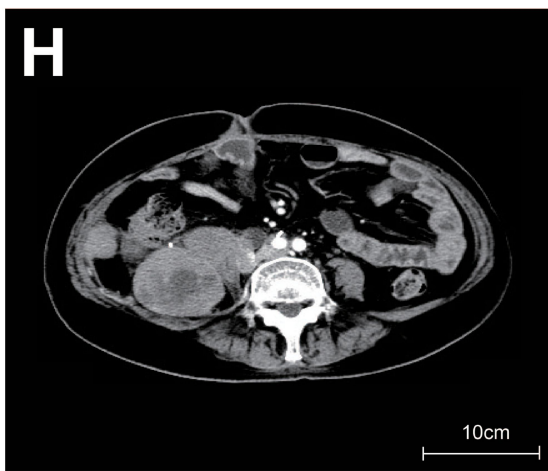

$02 / 12 / 2019$

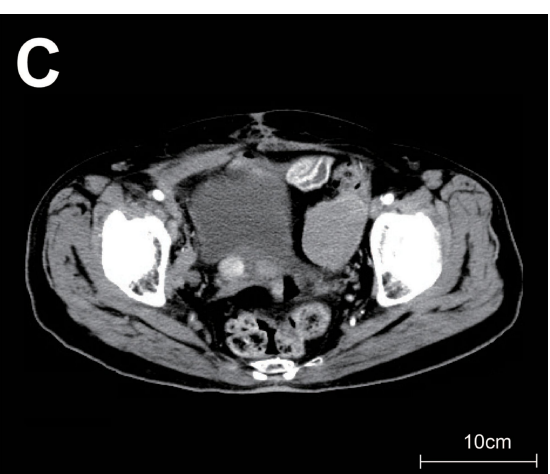

$07 / 18 / 2018$

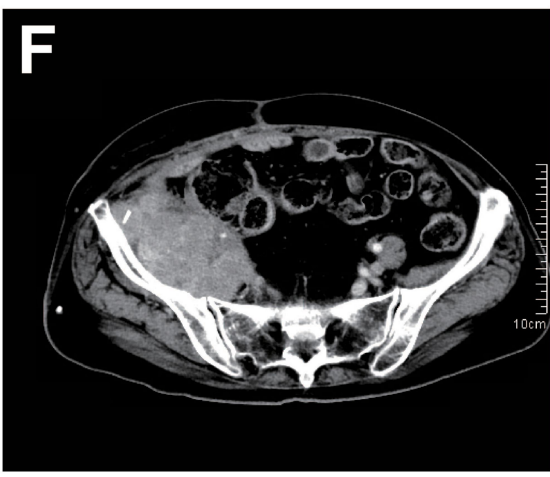

$11 / 30 / 2018$

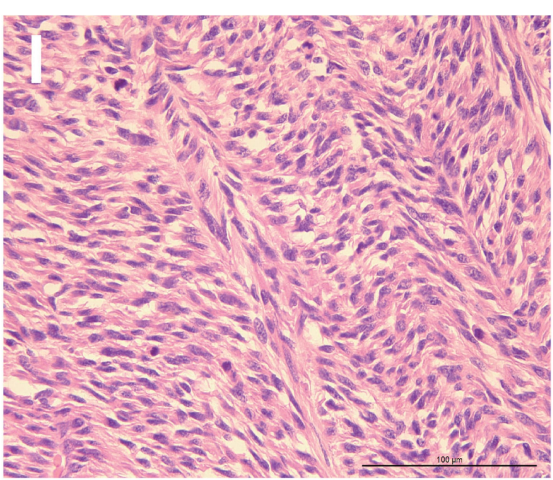

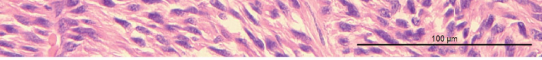

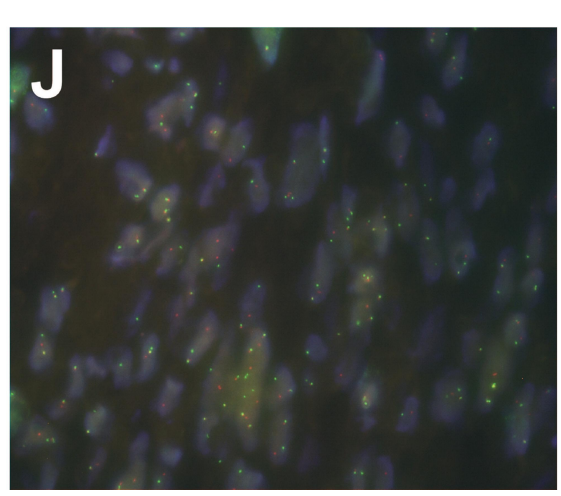

\section{K}

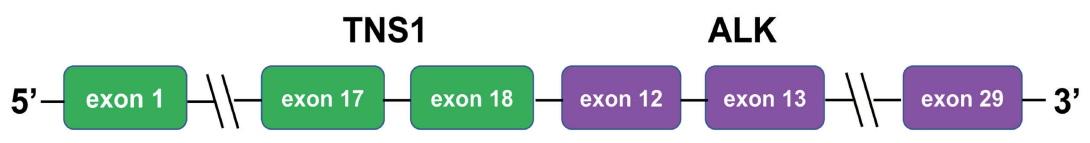

Figure 2 Radiological and histological evaluation and ALK translocation in patient 2. (A-H) Chronological records of CT images. The dates of the CTs were indicated below the images. (A) A mass of about $6.6 \times 9.6 \mathrm{~cm}$ in the pelvic cavity invading to the right ureter and right iliac vessels. (B and C) Multiple recurrences and metastases at the right abdominal cavity, pelvic cavity and abdominal wall region. (D and $\mathbf{E})$ The size of the pelvic tumor was reduced after crizotinib treatment. (F and $\mathbf{G})$ The lesions at the abdominal and pelvic cavity were stable after alectinib treatment. (H) The lesions in the abdominal cavity had progressed against alectinib treatment. (I) Micrograph of $\mathrm{HE}$ staining of the tumor biopsy at diagnosis (original magnification $\times 40$ ). (J) FISH image of the tumor biopsy labeled by an ALK probe (original magnification $\times 40$ ). (K) Diagram of ALK translocation. A 3 ' end fragment of ALK starting from exon 12 is fused behind TNSI exon 18. 
shrinkage (Figure 2D and E). At the end of September 2018, the lesions were stable although the patient still suffered severe pain in the right leg, indicating true progression, so another biopsy was taken and subjected to WES and RNA-Seq. The patient was switched to alectinib at $600 \mathrm{mg}$ b.i.d. on October 26, 2018. One month after that, the disease had been stabilized (Figure $2 \mathrm{~F}$ and $\mathrm{G})$. Unfortunately, the lesions in the abdominal cavity soon grew quickly as shown in the CT images of February 12, 2019 (Figure 2H), with the largest one reaching 10.7 $\mathrm{x} 7.17 \mathrm{~cm}$. Re-biopsy was taken and subjected to a third round of WES and RNA-seq analysis. Two genes, ESR1 and PTCH1, had significant reduction. A full list of genes with significant change of expression before and after resistance to crizotinib and alectinib are listed in Table 1.

\section{Discussion}

Sequencing analysis showed that patient 1 had an RRBP1ALK fusion. A similar fusion has been reported in an epithelioid inflammatory myofibroblastic sarcoma (EIMS) case with a poor prognosis. ${ }^{9,10}$ The recipient sites of ALK in both patient 1 and the reported EIMS patient were located in exon 20. However, the donating part of RRBP1 in the current patient is slightly different from the reported EIMS case. Patient 1 harbors exons 1-21 and a 34-bp piece of intron 21 of RRBP1, while the reported EIMS patient harbored exon 1-20 plus an alternatively spliced 33-bp intron. ${ }^{9}$ In either case, the coiledcoil domains of RRBP1 were preserved in the fusion protein which are predicted to promote self-dimerization, and ultimately facilitate the constitutive activation of the ALK kinase domain. This might explain the PR status of patient 1 after receiving crizotinib treatment as tumor cells still rely on ALK kinase activity to proliferate.

On the contrary, patient 2 presents a new TNS1-ALK fusion class. In a previous study, intron 16 of TNS1 was found fused to intron 19 of ALK in metastatic IMT patients. ${ }^{11}$ However, in patient 2 TNS1 exon 18 was fused to ALK exon 12. The created fusion protein in patient 2 still preserves an intact ALK kinase domain. Furthermore, exon 15 of TNS1 harbors a scaffold SH2 domain which can bind adaptor proteins and phosphotyrosine-containing proteins such as receptor tyrosine kinases (RTKs). SH2 domain is known to mediate dimerization of phosphorylated transcription factor STAT. ${ }^{12,13}$ Hence, we speculate that the fusion pattern in patient 2 may induce self-dimerization and activate the ALK kinase domain. Indeed, drug resistance quickly developed in patient 2
Table I Genes Differentially Expressed Before and After Drug Resistance Detected by RNA-Seq in Patient 2

\begin{tabular}{|l|l|l|l|}
\hline Gene & Sample & $\begin{array}{l}\text { Sample } \\
\text { B (Crizotinib } \\
\text { Resistance) }\end{array}$ & $\begin{array}{l}\text { Log } \text { Fold } \\
\text { Change (Sample } \\
\text { B/Sample A) }\end{array}$ \\
\hline CD274 & 0.419 & 2.400 & 2.518 \\
CDK6 & 25.361 & 2.749 & -3.206 \\
CHEKI & 1.290 & 5.228 & 2.019 \\
ESRI & 9.378 & 0.507 & -4.208 \\
IKZFI & 1.526 & 15.194 & 3.316 \\
KRAS & 2.579 & 0.578 & -2.158 \\
MLH3 & 10.661 & 1.157 & -3.203 \\
MYCN & 0.053 & 0.233 & 2.141 \\
PAX5 & 0.057 & 0.010 & -2.514 \\
PTCHI & 203.397 & 22.911 & -3.150 \\
SDHD & 3.630 & 0.419 & -3.115 \\
SOX2 & 0.110 & 0.014 & -2.979 \\
UGTIAI & 0.014 & 0.057 & 2.032 \\
WTI & 29.547 & 5.513 & -2.422 \\
\hline Gene & Sample & Sample & Log \\
& A (Operation) & C (Alectinib & Change (CIA) \\
& & Resistance) & \\
\hline AURKB & 10.968 & 2.463 & -2.155 \\
CTLA4 & 0.375 & 0.057 & -2.718 \\
CYPIA2 & 0.010 & 0.074 & 2.892 \\
ESRI & 9.378 & 0.861 & -3.445 \\
FLT3 & 0.233 & 0.026 & -3.162 \\
MYC & 4.460 & 0.267 & -4.062 \\
NTRKI & 2.830 & 0.419 & -2.755 \\
PTCHI & 203.397 & 40.973 & -2.312 \\
RET & 0.129 & 0.578 & 2.165 \\
SDHD & 3.630 & 24.775 & 2.771 \\
SMAD4 & 4.162 & 18.128 & 2.123 \\
U2AFI & 2.330 & 13.183 & 2.500 \\
\hline
\end{tabular}

with a PFS of only 2 months under crizotinib or alectinib treatment. This distinct TNS1-ALK fusion variant may represent a subtype of IMT with rapid clinical progression.

ALK inhibitors, including crizotinib, have been shown sensitive to a patient with ALK-translocated IMT. ${ }^{14}$ However, their efficacy can be limited by drug resistance. ${ }^{15}$ Acquired resistance to ALK inhibitors is often accompanied by three types of secondary mutations in the ALK kinase domain. ${ }^{16}$ The first and also the most common type involves disruption of the hydrogen bond network at the ALK tyrosine kinase domain. This group includes crizotinib-resisting L1196M and C1156Y mutations. Both L1196 and C1156 are the gatekeeping residues of the ALK kinase active site. L1196M would destabilize 
the hydrogen bond network in the A- and P-loop regions, and eventually affect the binding between ALK and crizotinib. ${ }^{17} \mathrm{C} 1156 \mathrm{Y}$ would disrupt the conformation of the ALK-binding pocket and also interfere with the hydrogen bonds there. ${ }^{18}$ The second ALK mutation type involves introduced steric hindrance at the drug-binding pocket. Those include G1269A, I1171T/N/S, G1202R, G1202del, D1203N, and S1206Y/C. ${ }^{19}$ The third ALK mutation type includes L1152R, I1151T-ins, and F1174C/L/V. They would change the affinity of the tyrosine kinase for ATP, thus enhance the enzymatic activity. ${ }^{20}$

Patient 1 experienced crizotinib resistance, postoperative progress and alectinib resistance. By comparing WES and RNA-Seq data between before crizotinib treatment and after alectinib resistance, we identified a new point mutation, L1196Q, in exon 23 in the ALK fragment with a mutation frequency of $1.06 \%$. RNA-seq analysis showed 96\% ALK transcripts encoded L1196Q. This mutation has been previously reported in human NPM-ALK anaplastic large cell lymphoma (ALCL) cell line, ${ }^{21}$ but is the first time reported in a clinical sample. The ALCL L1196Q cell line is resistant to crizotinib, probably because the substitution of 1196 side chain by glutamine resulted in a larger side chain that is energetically detrimental to crizotinib binding. Western blotting in the cell line showed increased level of phosphorylated ALK and STAT3, suggesting that the resistance was caused by ALK-mediated STAT3 activation. ${ }^{21}$ However, a report showed that ceritinib can overcome resistance to crizotinib caused by secondary mutations such as ALK G1296A. ${ }^{22}$ After receiving ceritinib, patient 1 achieved PR. This case demonstrated L1196Q mutation can also be overcome by ceritinib.

In addition to point mutations, gene amplification or ALK-independent mechanisms are other major causes of acquired resistance to ALK inhibitors. Amplification of EML4-ALK fusion gene or ALK copy number gain $(\mathrm{CNG})$ were both found in patients with $\mathrm{NSCLCs}^{23}$ and believed to induce resistance to crizotinib. EGFR and HER2 expression were found upregulated in tumors resistant to ALK inhibitors. ${ }^{24}$ Amplification of c-KIT, upregulated expression of PDGFRB, mutation of KRAS, and activation of MAPK, SRC and IGF1 pathways were all reported as potential causes of resistance in surveys. ${ }^{25}$ In conclusion, compensatory activation of bypass pathways is the basic principle of this type of resistance.

For patient 2, we collected samples at three time points, at the operation, after crizotinib resistance and after alectinib resistance. No any secondary mutation in the ALK fragment was identified, nor obvious copy number gain of ALK was observed (haploid copy number 2.48, 2.51 and 2.57, respectively, in WES analysis). ESR1 and PTCH1 are two genes with significant reduction of expression after the patient was resistant to crizotinib. Rearrangements of ESR1 can trigger endocrine resistance, and some hotspot ESR1 mutations can activate ER transcription, which was reported to promote hormone-independent tumor cell growth by ligand-independent ER activity in breast cancer. $^{26}$ However, in patient 2 the expression of ESR1 was decreased after drug resistance so could not explain the failure of the ALK inhibitors. On the other hand, PTCH1 has the highest FPKM value among all the genes with expression change, and the degree of its reduction almost reached one hundred folds. PTCH1 is a negative regulator of SHH signaling pathway, which is involved in oncogenesis of multiple cancers ${ }^{27}$ and is one of the downstream pathways activated by $\mathrm{ALK}^{28,29}$ Basal cell carcinoma (BCC) tumors had increased expression of ALK and the genes in SHH pathway. The expression of a key transcription factor of this pathway, glioma-associated oncogene homologue (GLI), was significantly reduced, and keratinocyte proliferation was inhibited after treatment with crizotinib in cell lines, suggesting a crucial role of ALK-mediated SHH signaling pathway in cell proliferation. ${ }^{30}$ Hence, we speculate that compensatory activation of the SHH signaling pathway due to $\mathrm{PTCH} 1$ reduction may lead to the resistance to ALK inhibitors in patient 2.

\section{Patient Update}

As of the manuscript date, patient 1 continues to receive ceritinib and is in good general condition without adverse drug reactions.

In August 2019, a family member of patient 2 was followed up on the phone. Patient 2, in poor financial condition, had insisted on taking alectinib until May 2019 since alectinib was free to her. Patient 2 passed away in June 2019.

\section{Ethics and Consent Statement}

This research was approved by the Ethics Committee of Zhongshan Hospital, Fudan University.

\section{Consent for Publication}

Written informed consents were obtained from the patients for publication of these cases and any accompanying images. 


\section{Funding}

This study was supported by Shanghai Sailing Program (19YF1407100).

\section{Disclosure}

Wending Sun, Jun Liu, Guan Wang, and Chun Dai are employees of GenomiCare Biotechnology Co. Ltd. The authors report no other conflicts of interest in this work.

\section{References}

1. Lovly CM, Gupta A, Lipson D, et al. Inflammatory myofibroblastic tumors harbor multiple potentially actionable kinase fusions. Cancer Discov. 2014;4(8):889-895. doi:10.1158/2159-8290.CD-14-0377

2. Pickett JL, Chou A, Andrici JA, et al. Inflammatory myofibroblastic tumors of the female genital tract are under-recognized: a low threshold for ALK immunohistochemistry is required. Am J Surg Pathol. 2017;41(10):1433. doi:10.1097/PAS.0000000000000909

3. Coffin CM, Patel A, Perkins S, Elenitoba-Johnson KS, Perlman E, Griffin CA. ALK1 and p80 expression and chromosomal rearrangements involving 2 p23 in inflammatory myofibroblastic tumor. Mod Pathol. 2001;14(6):569. doi:10.1038/modpathol.3880352

4. Shaw AT, Hsu PP, Awad MM, Engelman JA. Tyrosine kinase gene rearrangements in epithelial malignancies. Nat Rev Cancer. 2013;13 (11):772-787.

5. Debelenko LV, Arthur DC, Pack SD, Helman LJ, Schrump DS, Tsokos M. Identification of CARS-ALK fusion in primary and metastatic lesions of an inflammatory myofibroblastic tumor. Lab Invest. 2003;83(9):1255. doi:10.1097/01.LAB.0000088856.49388.EA

6. Mansfield AS, Murphy SJ, Harris FR, et al. Chromoplectic TPM3-ALK rearrangement in a patient with inflammatory myofibroblastic tumor who responded to ceritinib after progression on crizotinib. Ann Oncol. 2016;27(11):2111-2117. doi:10.1093/annonc/ mdw405

7. Palmer RH, Vernersson E, Grabbe C, Hallberg B. Anaplastic lymphoma kinase: signalling in development and disease. Biochem $J$. 2009;420(3):345-361.

8. Greenland C, Touriol C, Chevillard G, et al. Expression of the oncogenic NPM-ALK chimeric protein in human lymphoid T-cells inhibits drug-induced, but not Fas-induced apoptosis. Oncogene. 2001;20(50):7386. doi:10.1038/sj.onc. 1204870

9. Lee J-C, Li C-F, Huang H-Y, et al. ALK oncoproteins in atypical inflammatory myofibroblastic tumours: novel RRBP1-ALK fusions in epithelioid inflammatory myofibroblastic sarcoma. $J$ Pathol. 2017;241(3):316-323. doi:10.1002/path.4836

10. Mariño-Enríquez A, Wang W-L, Roy A, et al. Epithelioid inflammatory myofibroblastic sarcoma: an aggressive intra-abdominal variant of inflammatory myofibroblastic tumor with nuclear membrane or perinuclear ALK. Am J Surg Pathol. 2011;35(1):135-144. doi:10.1097/PAS.0b013e318200cfd5

11. Lee J, Singh A, Ali SM, Lin DI, Klempner SJ. TNS1-ALK fusion in a recurrent, metastatic uterine mesenchymal tumor originally diagnosed as leiomyosarcoma. Acta Med Acad. 2019;48(1):116-120.

12. Gopalasingam P, Quill L, Jeeves M, Overduin M. SH2 domain structures and interactions. In: SH Domains. Springer; 2015:159-185.

13. de Araujo ED, Orlova A, Neubauer HA, et al. Structural implications of STAT3 and STAT5 SH2 domain mutations. Cancers. 2019;11 (11):1757. doi:10.3390/cancers 11111757
14. Butrynski JE, D'Adamo DR, Hornick JL, et al. Crizotinib in ALK-rearranged inflammatory myofibroblastic tumor. $N$ Engl $J$ Med. 2010;363(18):1727-1733. doi:10.1056/NEJMoa1007056

15. Sasaki T, Okuda K, Zheng W, et al. The neuroblastoma-associated F1174L ALK mutation causes resistance to an ALK kinase inhibitor in ALK-translocated cancers. Cancer Res. 2010;70 (24):10038-10043. doi:10.1158/0008-5472.CAN-10-2956

16. Dagogo-Jack I, Shaw AT. Crizotinib resistance: implications for therapeutic strategies. Ann Oncol. 2016;27(Suppl 3):iii42-iii50. doi:10.1093/annonc/mdw305

17. Kay M, Dehghanian F. Exploring the crizotinib resistance mechanism of NSCLC with the L1196M mutation using molecular dynamics simulation. J Mol Model. 2017;23(11):323. doi:10.1007/s00894017-3495-5

18. Kumar A, Shanthi V, Ramanathan K. Computational investigation and experimental validation of crizotinib resistance conferred by C1156Y mutant anaplastic lymphoma kinase. Mol Inform. 2015;34 (2-3):105-114. doi:10.1002/minf.201400070

19. Nagasundaram N, Wilson Alphonse CR, Samuel Gnana PV, Rajaretinam RK. Molecular dynamics validation of crizotinib resistance to ALK mutations (L1196M and G1269A) and identification of specific inhibitors. $J$ Cell Biochem. 2017;118(10):3462-3471. doi:10.1002/jcb.26004

20. Lin JJ, Riely GJ, Shaw AT. Targeting ALK: precision medicine takes on drug resistance. Cancer Discov. 2017;7(2):137-155. doi:10.1158/ 2159-8290.CD-16-1123

21. Ceccon M, Mologni L, Bisson W, Scapozza L, Gambacorti-Passerini C. Crizotinib-resistant NPM-ALK mutants confer differential sensitivity to unrelated Alk inhibitors. Mol Cancer Res. 2013;11 (2):122-132. doi:10.1158/1541-7786.MCR-12-0569

22. Michels SYF, Scheel AH, Wündisch T, et al. ALKG1269A mutation as a potential mechanism of acquired resistance to crizotinib in an ALK-rearranged inflammatory myofibroblastic tumor. NPJ Precis Oncol. 2017;1(1):4. doi:10.1038/s41698-017-0004-3

23. Katayama R, Shaw AT, Khan TM, et al. Mechanisms of acquired crizotinib resistance in ALK-rearranged lung cancers. Sci Transl Med. 2012;4(120):120ra17-120ra17. doi:10.1126/scitranslmed.3003316

24. Wilson FH, Johannessen CM, Piccioni F, et al. A functional landscape of resistance to ALK inhibition in lung cancer. Cancer Cell. 2015;27(3):397-408. doi:10.1016/j.ccell.2015.02.005

25. Sharma GG, Mota I, Mologni L, Patrucco E, Gambacorti-Passerini C, Chiarle R. Tumor resistance against ALK targeted therapy-where it comes from and where it goes. Cancers. 2018;10(3):62.

26. De Santo I, Mccartney A, Migliaccio I, Leo AD, Malorni L. The emerging role of ESR1 mutations in luminal breast cancer as a prognostic and predictive biomarker of response to endocrine therapy. Cancers. 2019;11(12):1894. doi:10.3390/cancers11121894

27. Cortes JE, Gutzmer R, Kieran MW, Solomon JA. Hedgehog signaling inhibitors in solid and hematological cancers. Cancer Treat Rev. 2019;76:41-50. doi:10.1016/j.ctrv.2019.04.005

28. Lin JJ, Zhu VW, Yoda S, et al. Impact of EML4-ALK variant on resistance mechanisms and clinical outcomes in ALK-positive lung cancer. $J$ Clin Oncol. 2018;36(12):1199. doi:10.1200/ JCO.2017.76.2294

29. Della Corte CM, Viscardi G, Di Liello R, et al. Role and targeting of anaplastic lymphoma kinase in cancer. Mol Cancer. 2018;17(1):30. doi:10.1186/s12943-018-0776-2

30. Ning $\mathrm{H}$, Mitsui $\mathrm{H}$, Wang $\mathrm{CQ}$, et al. Identification of anaplastic lymphoma kinase as a potential therapeutic target in basal cell carcinoma. Oncotarget. 2013;4(12):2237. 


\section{Publish your work in this journal}

OncoTargets and Therapy is an international, peer-reviewed, open access journal focusing on the pathological basis of all cancers, potential targets for therapy and treatment protocols employed to improve the management of cancer patients. The journal also focuses on the impact of management programs and new therapeutic agents and protocols on patient perspectives such as quality of life, adherence and satisfaction. The manuscript management system is completely online and includes a very quick and fair peer-review system, which is all easy to use. Visit http://www.dovepress.com/ testimonials.php to read real quotes from published authors.

Submit your manuscript here: https:/www.dovepress.com/oncotargets-and-therapy-journal 